\title{
Aggregation State-Permeation Characteristic Relationships of Self-Supported Liquid Crystalline Membranes
}

\author{
Tisato Kajiyama, ${ }^{*}$ Atsushi Takahara, and Hirotsugu KiKuchi \\ Department of Applied Chemistry, Faculty of Engineering, \\ Kyushu University, 6-10-1, Hakozaki, Higashi-ku, Fukuoka 812, Japan
}

(Received November 26, 1990)

\begin{abstract}
A series of built-up films composed of the blend materials of polymer and liquid crystal(LC) were prepared by a solvent-casting or a water-casting method. The weight fraction of LC in the composite membrane was $60-70 \%$. The thickness and aggregation state in the composite membrane can be controlled by solvents and the concentration of a solution. LC material forms a continuous phase in the three-dimensional spongy network of the matrix polymer. Therefore, LC phase can play a role of a diffusing phase for the permeants, because of very low viscosity of LC. The composite membranes were applied as the permselective membranes for the purpose of oxygen enrichment, molecular filtration, active transports of metal cations and enantioselective transport of amino acid salts.

KEY WORDS Polymer-(Liquid Crystal) Composite Membrane I Self-supported Liquid Crystal / Oxygen Enrichment I Molecular Filtration / Active Transport / Photo-responsive / Optical Resolution /
\end{abstract}

The permeation of gases and liquids through polymer films has been studied by investigating the chemical nature, aggregation state, and thermal molecular motion of matrix polymeric chains. Biological membranes are composed of various types of phospholipids, cholesterols and functional proteins. Bimolecular membrane of lipids are in a liquid crystalline state, capable of reversible structural modifications, and permeation properties of biomembranes depend upon such reversible changes. For application to practical membrane systems without losing this essential controlling function, liquid crystalline compounds would be useful instead of phospholipids because they are much more stable than phospholipids in aqueous systems and show a similar gel-liquid crystal phase transition phenomenon.

A polymer/(liquid crystal: LC) composite membrane is a blend thin film cast from a polymer solution with nematic liquid crystalline materials. ${ }^{1-4}$ Characteristics of nematic liquid crystalline materials include low viscosity comparable to water, and orientation of molecules in the presence of an electric field. 
A polymer/LC composite membrane can control solubility and diffusivity of the permeants with their chemical structures and/or excellent fluidity of liquid crystalline material. For a composite membrane composed of mechanically tough matrix polymer, an ultrathin and fairly wide-area membrane is expected, in spite of the fact that the characteristics of this system are analogous to a liquid membrane. ${ }^{5-7}$ The purpose of this study is to investigate the structural properties or compatibilities of the composite membrane composed of polymer and liquid crystalline material, the thermal molecular motion-permeation relationship, and also, the permselective mechanism of the composite membrane to hydrocarbon gases, oxygen and nitrogen or, cationic ions or D-and L-amino acid salts. Electric field and photoresponsive effects on permeation have also been studied.

\section{EXPERIMENTAL}

Figure 1 shows the chemical structures of matrix polymers and liquid crystalline materials for the composite membrane. TKN and $\mathrm{T}_{\mathrm{N}}$ are the phase transition temperatures from crystal to nematic and from nematic to isotropic, respectively. The polymer/LC composite membranes contain liquid crystal(LC) of $15,30,45$, and $60 \mathrm{wt} \%$. These composite membranes are designated the 85/15, $70 / 30,55 / 45$, and $40 / 60$ composites, respectively.

In order to study oxygen permselective characteristics, the polymer/LC/(fluorocarbon monomer:FC) ternary composite membrane was prepared from a solution mixture. Perfluorotributylamine(PFTA) and Tris $(1 \mathrm{H}, 1 \mathrm{H}, 5 \mathrm{H}$ octafluoropentyl)phosphate(TPP) were chosen as the FC monomers, because they have both an excellent oxygen solubility

1. POLYMER

1) Polycarbonate(PC)

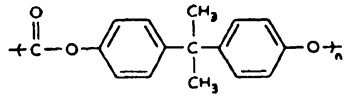

2) Poly(vinyl chloride)(PVC)<smiles></smiles>

2. LIQUID CRYSTAL

1) N-(4-ethoxybenzyl idene) - - ''$\mathrm{CH}_{3} \mathrm{CH}_{2} \mathrm{O}-\mathrm{O}-\mathrm{CH}=\mathrm{N}-\mathrm{O}-\left(\mathrm{CH}_{2}\right)_{3} \mathrm{CH}_{3}$

2) 4-cyano-4' -pentyl biphenyl (CPB)

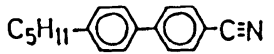

$K \rightarrow N 296 K, N \rightarrow I 308 K$

3) 4-cyano-4'-heptyloxy bipheny 1(CHOB)

$\mathrm{C}_{7} \mathrm{H}_{15} \mathrm{O}-\mathrm{O}-\mathrm{O}-\mathrm{O} \equiv \mathrm{N}$

$K \rightarrow H 25 K, N \rightarrow I 341 K$
3. FLUOROCARBON MONOMER

1) Perfluorotributylamine(PFTA) $\left(\mathrm{CF}_{3} \mathrm{CF}_{2} \mathrm{CF}_{2} \mathrm{CF}_{2}\right)_{3} \mathrm{~N}$

2) $\operatorname{Tris}(1 \mathrm{H}, 1 \mathrm{H}, 5 \mathrm{H}$-octafluoropentyl)

$\left(\mathrm{HCF}_{2} \mathrm{CF}_{2} \mathrm{CF}_{2} \mathrm{CF}_{2} \mathrm{CH}_{2} \mathrm{O}\right)_{3} \mathrm{PO}$

4. PHOTORESPONSIVE CROWN ETHER(AZO-CR)

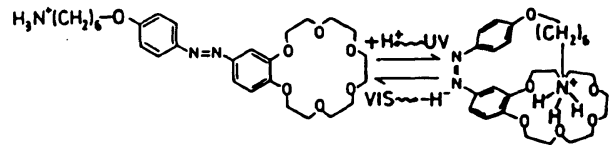

5. CHIRAL CROWN ETHER(CR*)
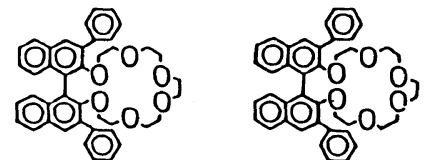

(R) $-C R^{*} 81.8 \%$

(S) $-C R^{*} 98.2 \%$

Figure 1. Chemical structures of components for a polymer/LC composite membrane. 
(for example, $0.43 \mathrm{~cm}^{3} \mathrm{O}_{2} / \mathrm{cm}^{3}$ PFTA at $310 \mathrm{~K}$ ) and a high thermal stability in comparison with other FC monomers. The weight ratio of each component was PVC/EBBA/FC/L44 $=40 / 60 / 7.2 / 7.2$. The molar ratio of LC/PFTA was 20/1.

To investigate photoresponsive active transport of $\mathrm{K}^{+}$through the composite film, the ternary composite thin film was prepared by the water-cast method: a solution of PVC, EBBA and crown ether bonded by an azobenzene group(AZO-CR) dissolved in a suitable solvent was poured onto the water surface at $283 \mathrm{~K}$ and allowed to spread. An ultrathin film was formed on the water surface after evaporation of the solvent. The weight ratio of PVC/EBBA/AZO-CR was 40/60/5. The $30-35$ layers of the watercast membrane, of $-50 \mathrm{~nm}$ thick, were built-up to prepare the thin film.

The PVC/EBBAVR* ternary composite membrane was prepared by water-cast method for the purpose of optical resolution. A mixture of THF/toluene (1/2 wt/wt) was used as a water-cast solvent. The permeability to $D$ - and L-phenylglycine perchlolate was evaluated by a normal osmosis method through the composite thin film stacked with water-casting membranes of 30 layers.

\section{RESULTS AND DISCUSSION}

\section{Aggregation State of Polymer/LC Composite Membrane}

Figure 2 shows the

scanning electron

microscopic (SEM)

photographs of the fracture

surface (upper portion in

each photograph) and

membrane one (lower

portion) for the $40 / 60$

(PVC/EBBA) composite

membrane after extracting EBBA with ethanol at $333 \mathrm{~K}$ for $2 \mathrm{hr}$. AFS and SFS represent the air-facing surface and the substratefacing one, respectively, when the membrane was cast. The sample presents an appearance of spongy networks of PVC material. Since about $95 \mathrm{wt} \%$ of total EBBA was extracted from the composite membrane with hot ethanol, it is evident that EBBA

molecules form domains as

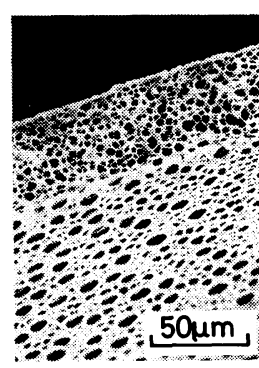

FSIAFS

(a)

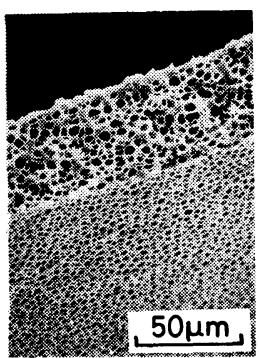

FS/SFS

(b)

Figure 2. Scanning electron micrographs of fracture surface (FS: upper portion in each photograph) and membrane surface ((a) AFS and (b) SFS: lower portion) for $40 / 60$ (PVC/EBBA) composite membrane after extracting EBBA with ethanol at $333 \mathrm{~K}$ for $2 \mathrm{hr}$.

an interpenetrating continuous phase among the 3-dimensional spongy networks of PVC matrix. Consequently, it seems reasonable to consider that the continuous EBBA phase takes a role of main diffusing region for gas, ion or carriers. In addition, the concentration of EBBA on SFS was relatively a little higher than that on AFS due to higher hydrophobic character of LC. Thus, 
the gas permeation experiments in this study were consistently performed by use of the AFS side as a gas feed surface. 5

Furthermore, the formation process of the composite membrane was traced with a polarizing optical microscope(POM). Liquid crystalline molecules were gradually clustering or aggregating with a solvent evaporation, resulting in an exclusion of PVC molecules. At a certain concentration, the crystallite-like domains of EBBA were produced and began to grow in PVC matrix. Simultaneously, an excluded PVC phase surrounded EBBA crystal domains as the networks in a similar manner to a coacervation effect. Consequently, distinct crystal domains of EBBA were formed in the composite membrane as a continuous phase and the characteristic interpenetrated structure composed of both components was completed in this process.

Ultrathin membranes were prepared by careful spreading a single drop of a solution of the mixed phases on the water surface. The aggregation state and the thickness of the ultrathin membrane were affected with casting conditions such as the kind of solvent, the concentration of a solution and the temperature of the water. The PVC matrix is remarkably porous when the weight ratio of THF/toluene is $1 / 2$ and also, the concentration of solution ranges 8-10 wt \% . Since the original thin membrane are nonporous before extracting EBBA, the porous portions surrounded by $P V C$ fibrils must be filled with EBBA before extraction. Since EBBA exists as a continuous domain penetrating throughout the composite membrane, the EBBA phase can serve as a transporting or diffusing phase for premeates or carriers.10-16

2. Permeation Mechanism of Polymer/LC (40/60) Composite Membrane

The aggregation state of the components in the composite membrane was investigated on the basis of sorption characteristics of gases such as sorption isotherms or sorptiondesorption experiments. The measurements were carried out above and below TKN of LC. The validity of Henry's law for the sorption isotherm of these hydrocarbon gases below $\mathrm{T}_{\mathrm{KN}}$ is doubtful, but above ${ }_{\mathrm{KN}}$ it obeys the law. Figure 3 exhibits the

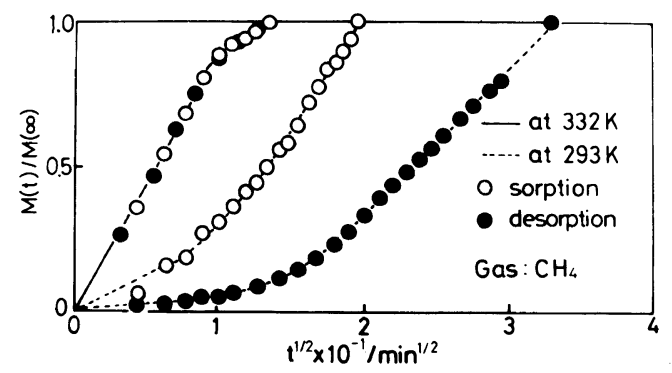

Figure 3. Sorption-desorption curves for $\mathrm{CH}_{4}$ in the $40 / 60$ (PC/EBBA) composite membrane at $293\left(<T_{K N}\right)$ and $332 \mathrm{~K}\left(>\mathrm{T}_{\mathrm{KN}}\right)$. sorption-desorption curves of $\mathrm{CH}_{4}$ in the $40 / 60$ (PC/EBBA) composite membrane. Here, $M(t)$ is the quantity of penetrant which has entered or left the membrane in time $t$, and $M(\infty)$ is the corresponding quantity at infinite time. The linear ralationship between $M(t) / M(\infty)$ and $t^{1 / 2}$ was recognized in the early stage of an experiment at $332 \mathrm{~K}$ (above $\mathrm{T}_{\mathrm{KN}}$ ), and this shape of the $M(t) / M(\infty)$ curve suggests that the system follows Fickian sorption. Therefore, in the temperature range above $\mathrm{T}_{\mathrm{KN}}$, it is reasonable to consider that a steady surface equilibrium is 
established immediately, and that the diffusion coefficient of $\mathrm{CH}_{4}$ is a function of the concentration only. Similar behavior was observed for the polymer-organic vapor system in the rubbery state The sorption or desorption experiments apparently indicate that the $40 / 60$ (PC/EBBA) composite membrane can be handled as a homogeneous medium when considering gas permeation is carried out in the temperature range above $\mathrm{T}_{\mathrm{KN}} \cdot 2-4$

Figure 4 shows the Arrhenius plot of the permeability coefficients, $\mathrm{P}$, to $\mathrm{He}, \mathrm{N}_{2}$ and various hydrocarbon gases for the $40 / 60$ (PC/EBBA) composite membrane. The distinct jump of $P$ was observed in the vicinity of $T_{K N}$ of EBBA. In particular, the magnitude of $P$ to hydrocarbon gases increases by approximately 100-500 times over a few degrees in the phase transition region of EBBA ( $T_{K N}$ ) shown by broken lines in Figure $4 . \quad$ In this region, permeabilities of $\mathrm{He}$ and $\mathrm{N}_{2}$ gases also increase remarkably. As $\mathrm{He}$ gas is inert, its solubility property to the composite membrane may not change over the temperature range of the phase transition. Therefore, this discontinuous increase of $P$ for inert gas may be caused by a remarkable increase of diffusion coefficient. With respect to permeation of various hydrocarbon gases, the magnitudes of $\mathrm{P}$ increase in the order $\mathrm{i}-\mathrm{C}_{4} \mathrm{H}_{10}, \mathrm{C}_{4} \mathrm{H}_{10}, \mathrm{C}_{3} \mathrm{H}_{8}$ and $\mathrm{CH}_{4}$ in the temperature range

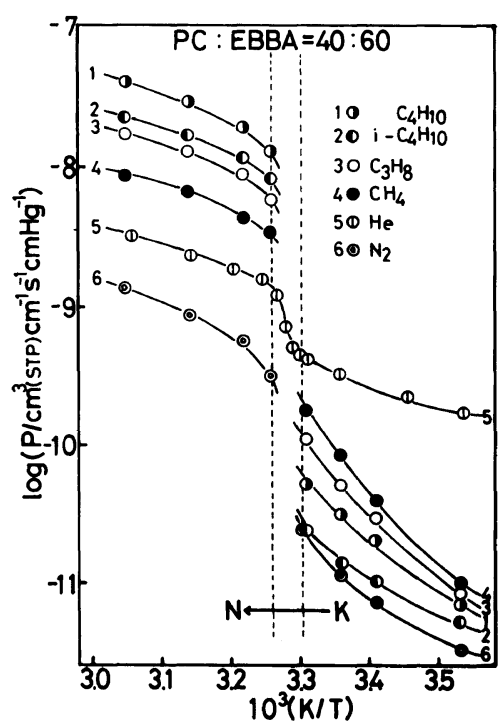

Figure 4. Arrhenius plot of $\mathrm{P}$ of $\mathrm{He}, \mathrm{N}_{2}$, and various hydrocarbon gases for 40/60 (PC/EBBA) composite membrane. (1) $\mathrm{C}_{4} \mathrm{H}_{10}$, (2)i$\mathrm{C}_{4} \mathrm{H}_{10}, \quad(3) \mathrm{C}_{3} \mathrm{H}_{8}, \quad$ (4) $\mathrm{CH}_{4}$, (5) $\mathrm{He}, \quad(6) \mathrm{N}_{2}$

below TKN and in the order $\mathrm{CH}_{4}, \mathrm{C}_{3} \mathrm{H}_{8}, \mathrm{i}-\mathrm{C}_{4} \mathrm{H}_{10}$, and $\mathrm{C}_{4} \mathrm{H}_{10}$ in the temperature range above the transition. The variations of $P$ with temperature to $\mathrm{CH}_{4}, \mathrm{C}_{3} \mathrm{H}_{8}$ and $\mathrm{C}_{4} \mathrm{H}_{1}$ are nearly reversible across the phase transition range. This trend may suggest that the permeation process is predominantly governed by the diffusion process. On the other hand, above TKN of EBBA, the magnitudes of $P$ increase with increasing number of carbon atoms. Generally speaking, this tendency cannot be explained by the diffusion control mechanism, but it is reasonably expected that permeability is governed mainly by the solubility characteristic. ${ }^{4}$

3. Oxygen Enrichment through Polymer/LC/Fluorocarbon Ternary Composite Membrane

Figure 5 shows the plots of $\mathrm{P}\left(\mathrm{O}_{2}\right) / \mathrm{P}\left(\mathrm{N}_{2}\right)$ against $\mathrm{P}\left(\mathrm{O}_{2}\right)$ for the FCcontained (curves 1 and 2) and $F C$-free (curve 3 ) composite membranes, including the gas separation data for ordinary polymers. An increase in $\mathrm{P}\left(\mathrm{O}_{2}\right)$ corresponds to an increase of the measuring temperature. The permeability coefficient ratio of the PFTA-contained composite membrane showed the maximum just above $T_{K N}\left(P\left(\mathrm{O}_{2}\right) / P\left(N_{2}\right)=5.10\right)$. This value was about 
two times greater than that for the PFTA-free one. The temperature dependence of the solubility coefficient in the polymer/LC composite membrane reaches its maximum around $\mathrm{T} K \mathrm{KN}$. The order of magnitude of $P$ for the PFTA-contained composite membrane was about $10^{-9}$ to $10^{-8} \mathrm{~cm}^{3}$ (STP) $\mathrm{cm}^{-1} \mathrm{~s}^{-1} \mathrm{cmHg}^{-1}$ and the permeability coefficient ratio was about $3.5-4.0$ in a nematic or isotropic state of EBBA . Furthermore, above $\mathrm{Tg}$ of PVC in the composite membranes (shown by an arrow in Figure 5), the FCcontained composite membrane as well as the FC-free one had a unique effect on the $\mathrm{P}\left(\mathrm{O}_{2}\right) / \mathrm{P}\left(\mathrm{N}_{2}\right)-\mathrm{P}\left(\mathrm{O}_{2}\right)$ relationship; the magnitudes of $\mathrm{P}\left(\mathrm{O}_{2}\right) / \mathrm{P}\left(\mathrm{N}_{2}\right)$ increased with increasing $\mathrm{P}\left(\mathrm{O}_{2}\right) .{ }^{10}$ This contrasts behavior of ordinary polymeric membranes for gas separation; $\mathrm{P}\left(\mathrm{O}_{2}\right) / \mathrm{P}\left(\mathrm{N}_{2}\right)$ generally decreases with an increase in $\mathrm{P}\left(\mathrm{O}_{2}\right)$. Although the mechanism for this unique behavior has not yet been clarified, we speculate the reason for the FC-free composite membrane is that the frequency with which the polymeric chains make direct contact with oxygen and nitrogen gases on the upstream surface is considerably increased by the activated thermal molecular motion of matrix PVC chains above Tg. In this case, the much greater oxygen selectivity of PVC over liquid crystalline material may contribute to improving permeation characteristics above Tg. Furthermore, in the case of the FC-contained composite membrane, this unique effect is deduced from properties of FC monomers. Although the solubility of both oxygen and nitrogen gases in FC monomers decreases with increasing temperature, a decrease to oxygen is less than nitrogen. Consequently, this effect may contribute to an increase of $\mathrm{P}\left(\mathrm{O}_{2}\right) / \mathrm{P}\left(\mathrm{N}_{2}\right)$, despite the continuous increase in $\mathrm{P}\left(\mathrm{O}_{2}\right)$ above $\mathrm{Tg}$ of PVC.

\section{Molecular Filtration through Polymer/LC Composite Membrane}

The polymer/LC composite membranes were cast from a 1,2dichloroethane solution of PVC and 4-cyano-4'-pentylbiphenyl (CPB) or 4-cyano-4'-heptyloxybiphenyl(CHOB) which have the positive dielectric anisotropy, by using the homemade casting apparatus. When an electric fields is applied during evaporation of solvent, $\mathrm{CPB}$ or $\mathrm{CHOB}$ molecules are preferentially oriented in the direction of an electric field. A pair of electrodes was set in the permeation cell. Therefore, an electric field could be applied during measurements of gas permeation. The space between the electrodes was $5 \mathrm{~mm}$. When an electric field was applied perpendicular to the membrane surface, it emphasizes the normal alignment to the film surface. Since LC molecules aggregate as a continuous phase in the composite membrane, it may be possible to control diffusivity of gas molecules through the composite 
membrane under application of an electric field, on the basis of the relationship between the dimension of channel formed in the intermolecular regions and the sectional dimensions of permeating gases.

Figure 6 shows the applied voltage dependence of $P$ for $\mathrm{C}_{4} \mathrm{H}_{10}$ and iso- $\mathrm{C}_{4} \mathrm{H}_{10}$ gases. The diameter difference of the section for $\mathrm{C}_{4} \mathrm{H}_{10}$ and iso- $\mathrm{C}_{4} \mathrm{H}_{10}$ is only 0.07 $\mathrm{nm}\left(\mathrm{dc}_{4} \mathrm{H}_{10}=0.49 \mathrm{~nm}\right.$ and

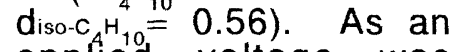
applied voltage was increased, the magnitudes of $P$ for both $\mathrm{C}_{4} \mathrm{H}_{10}$ and iso$\mathrm{C}_{4} \mathrm{H}_{10}$ gradually increassed. The tendency on $P$ with an applied voltage can be explained by the relative difference of diffusion path that gas molecules diffuse along a fairly straight

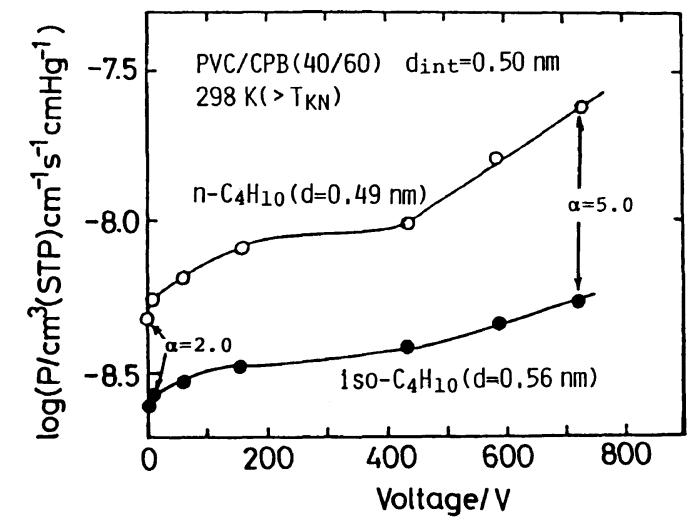

Figure 6. The variation of permeability coefficients for $\mathrm{C}_{4} \mathrm{H}_{10}$ and iso- $\mathrm{C}_{4} \mathrm{H}_{10}$ through the 40/60 (PVC/CPB) composite membrane with the magnitude of applied voltage.

path in the case of the oriented composite membrane and on theother hand, they do along a tortuous path in the case of the unoriented membrane. The separation ratio of $\mathrm{P}\left(\mathrm{C}_{4} \mathrm{H}_{10}\right)$ to $\mathrm{P}$ (iso- $\mathrm{C}_{4} \mathrm{H}_{10}$ ) $\alpha$ was 2 at zero (random orientation of LC molecules), and increased to 5 at 730 volt(oriented state). An increase of $\alpha$ with the magnitude of an applied voltage indicates that the distribution of channel dimension existing among LC molecules becomes narrower due to more progressed molecular orientation perpendicular to the membrane surface. This result indicates that the greater degree of orientation of LC molecules induces more effective separation between $\mathrm{C}_{4} \mathrm{H}_{10}$ and iso- $\mathrm{C}_{4} \mathrm{H}_{10}$, even though their diameter difference of molecular sections is smaller than $0.1 \mathrm{~nm} .4,8,11$

5. Photoresponsive Active Transport of Metal Cations through Polymer/LC/(Crown Ether) Ternary Composite Membrane

Photo-induced active transfer of $\mathrm{K}^{+}$for the polymer/LC/AZO-CR ternary composite membrane is possible. This ternary composite thin film was prepared using the water-cast method. A solution of PVC, EBBA and AZO-CR dissolved in a mixed solvent of THF, toluene and chloroform(solution concentration of $8 \mathrm{wt} \%$ ) was dropped onto the water surface at $283 \mathrm{~K}$. The weight -ratios of PVC/EBBA/AZO-CR and THF/toluene/chloroform are 40/60/4 and $2 / 4 / 1$, respectively. $30-50$ layers of the water-cast membrane, approximately $50 \mathrm{~nm}$ thick were built-up to form the thin film. The trans-cis photoisomerization of $A Z O-C R$ in the composite thin film was confirmed by the UV or VIS light. The permeation experiment was carried out using a permeation cell attached to an irradiation system of VIS and UV light sources. Basic and acidic 
aqueous phases with potassium p-toluenesulfonate of $1 \times 10^{-4} \mathrm{M}$ were separated by the ternary composite thin film. The VIS and UV light sources were arranged to irradiate perpendicular to the film surface from basic and acidic aqueous phases, respectively . The $\mathrm{K}^{+}$transport experiment was carried out at $313 \mathrm{~K}(\mathrm{a}$ temperature above $T_{K N}$ of EBBA). The concentration variation of $\mathrm{K}^{+}$was evaluated by atomic absorption spectrophotometry.

Figure 7 shows the time dependence of the amount of $\mathrm{K}^{+}$transported from the basic aqueous phase to the acidic phase upon photoirradiation and also, in the dark. Both the acidic and basic aqueous phases contained the same concentration of $\mathrm{K}^{+}$before permeation experiment started. Since there are two driving forces, photoirradiation and $\mathrm{pH}$ gradient(proton gradient), we have to evaluate the magnitudes of $\mathrm{K}^{+}$permeation upon photoirradiation and in the dark, separately, in order to discuss a photoresponsive active transport of $\mathrm{K}^{+}$. It is clear that the permeation rate of $\mathrm{K}^{+}$upon photoirradiation is greater than the rate in the dark as

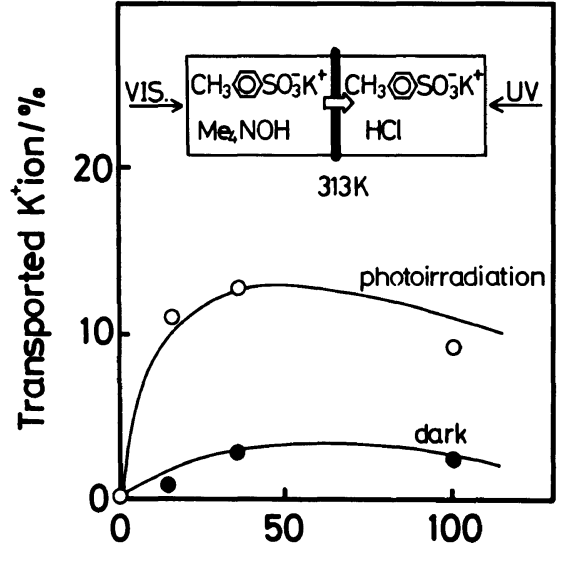

Time/min.

Figure 7. Time dependence of the relative amount of transported $\mathrm{K}^{+}$into an acid aqueous phase upon (1) photoirradiation and (2) in the dark.

shown in Figure 7 . This indicates that an active transport of $\mathrm{K}^{+}$ takes place from the basic aqueous phase to the acidic one through the ternary composite thin film upon photoirradiation. This is the first report to show a photo-induced active transport of metal cation through an organic thin film.

Figure 8 shows the schematic representation of the permeation mechanism of $\mathrm{K}^{+}$through the PVC/EBBA/AZO-CR ternary composite thin film upon photoirradiation. At the surface of the composite thin film contacting the basic aqueous phase, trans-AZO-CR exists preferentially upon irradiation with VIS light. Trans-AZO-CR easily forms a complex with $\mathrm{K}^{+}$and trans-AZO-CR entrapping $\mathrm{K}^{+}$ diffuses to the opposite side of the film contacting the acid aqueous phase owing to the concentration gradient of the transisomer in the ternary composite thin film. At the surface contacting the acid aqueous phase, an amino tail group of AZO-CR is isomerized to the cis-isomer upon irradiation with UV light, the crown ether ring binds intramolecularly to the protonated ammonium group, so that cis-AZO-CR effectively releases $\mathrm{K}^{+}$into the acidic aqueous phase. Furthermore, cis-AZO-CR returns to the side of the basic aqueous phase owing to the concentration gradient of the cis-isomer in the ternary composite thin film and again, is isomerized to the trans-isomer upon irradiation with VIS light or heating. Since protonated cis-AZO-CR becomes hydrophilic, the composite film surface contacting the acidic aqueous phase was coated with a plasticized thin PVC membrane of $60 \mathrm{~nm}$ thick in order to prevent leakage of protonated-AZO-CR 


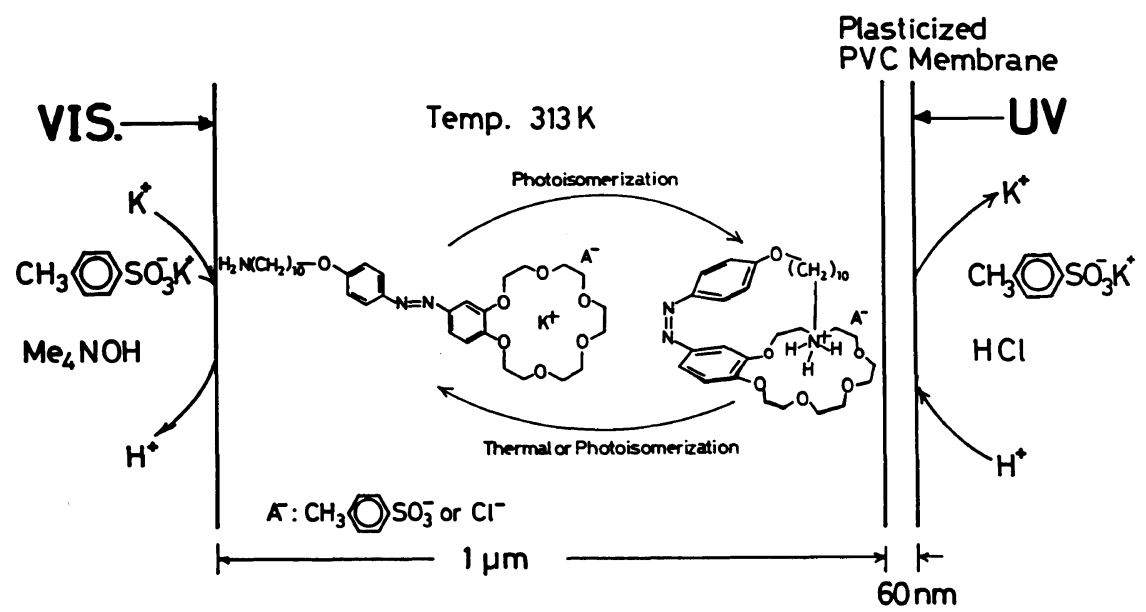

\section{Basic aqueous Composite Membrane phase \\ Acidic aqueous phase}

Figure 8. Schematic representation of the $\mathrm{K}^{+}$permeation mechanism through the ternary composite thin film upon photoirradiation.

into the acidic aqueous phase. $8,9,12$,

6. Optical Resolution of Amino Acid Salts through Polymer/LC/CR* Composite Thin Membrane

The polymer ILC composite film was prepared as a wide area ultra thin film of $20 \mathrm{~nm}$ thick by using the water-cast method. In the case of carrier-facilitated transport, a thinner film is more advantageous for the purpose of perchlolate by enantio selective transport was investigated by using the ternary composite thin film.

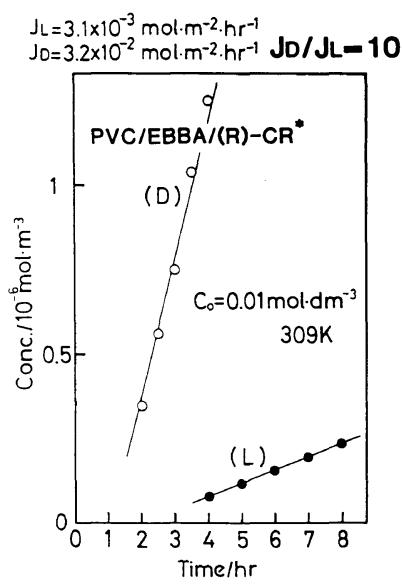

(a)

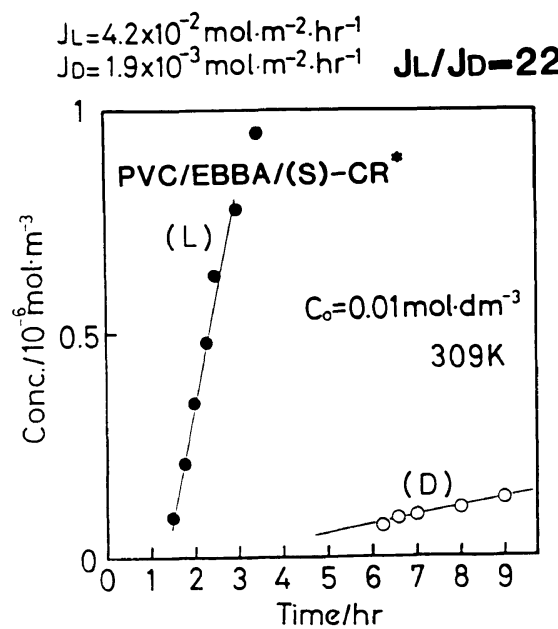

(b)

Figure 9. Time dependence of concentration variations of $D$ - and L-phenylglycine perchlolate through the $\mathrm{PVC} / \mathrm{EBBA} /(\mathrm{R})-\mathrm{CR}^{*}$ $(40 / 60 / 0.5)$ (a) and $\mathrm{PVC} / \mathrm{EBBA} /(\mathrm{S})-\mathrm{CR}^{*}$ (40/60/2) (b) composite films at $309 \mathrm{~K}$. 
Figure 9 shows the time dependence of the transported amount of $D$ - or L-phenylglycine perchlolate through the polymer/LC/CR* ternary composite thin film. High enantio selective transport was observed. The flux ratio of each enantiomer of phenylglycine perchlolate, $J_{D} / J_{L}$ or $J_{L} / J_{D}$ was $10-20$. The polymer/LC/CR*, ternary composite thin film can be a promising novel membrane for a optical resolution system exhibiting high enantio selectivity and large flux. Furthermore, it is suggested that the chiral helical field composed of nematic molecular assembly, induced by addition of $\mathrm{CR}^{\star}$, must also fairly enhance optical resolution. We believe that the induced chiral nematic phase accelerates the ability of optical resolution through the composite thin film.14,15

\section{REFERENCES}

1. T. Kajiyama, Y. Nagata, E. Maemura and M. Takayanagi, Chem. Lett., 679(1979).

2. T. Kajiyama, Y. Nagata, S. Washizu and M. Takayanagi, J. Membrane Sci., 11, 39 (1982).

3. T. Kajiyama, S. Washizu and M. Takayanagi, J. Appl. Polym. Sci., 29, 3955 (1984).

4. T. Kajiyama, S. Washizu, A. Kumano, I. Terada and M. Takayanagi, J. Appl. Polym. Sci., Appl. Polym. Symp., 41, 327(1985).

5. T. Kajiyama, H. Kikuchi, M. Katayose and S. Shinkai, New Polym. Mater., 1, 99 (1988).

6. T. Kajiyama, MRS Int'l. Mtg. on Adv. Mats., 1, 241 (1989).

7. S. Shinkai, K. Shimamoto, S. Nakamura, O. Manabe, and T. Kajiyama, J. Polym. Sci., Polym. Lett., 25, 495 (1987).

8. T. Kajiyama, H. Kikuchi, I. Terada, M. Katayose, A. Takahara and S. Shinkai, Current Topics Polym. Sci.-1984, 2, 315 (1987).

9. T. Kajiyama, H. Kikuchi and S. Shinkai, J. Membrane Sci., 36, 243 (1988).

10. T. Kajiyama, S. Washizu and Y. Ohomori, J. Membrane Sci., 24, 73 (1985).

11. T. Kajiyama, J. Macromol. Sci., Chem., A25, 583 (1988).

12. T. Kajiyama, H. Kikuchi, I. Terada, M. Katayose, and A. Takahara, Current Topics in Polym. Sci., 2, 320 (1985).

13. H. Kikuchi, M. Katayose. S. Shinkai, O. Manabe and T. Kajiyama, J. Chem. Soc. Jpn., Chem. Ind. Chem., 432 (1987).

14. T. Kajiyama, H, Kikuchi and A. Miyamoto, "Frontiers of Molecular Science", IUPAC505 (1989).

15. H. Kikuchi, J. Hattori, Y. Mori, and T. Kajiyama, Kagaku Kogaku Ronbunshu, 15, 617(1989). 